\title{
Local Assessment of Statokinesigram Dynamics in Time: An in-Depth Look at the Scoring Algorithm
}

\author{
Ioannis Bargiotas ${ }^{1,2}$, Julien Audiffren ${ }^{1,2 *}$, Nicolas Vayatis ${ }^{1,2}$, Pierre-Paul Vidal ${ }^{2}$, \\ Alain P. Yelnik ${ }^{2,3}$, Damien Ricard ${ }^{2,4,5}$. \\ 1 CMLA, ENS Paris-Saclay, Université Paris-Saclay, Cachan, France \\ 2 COGNAG-G UMR 8257, CNRS, SSA, Université Paris Descartes, Paris, France \\ 3 PRM Department, GH Lariboisière F. Widal, AP-HP, Paris Diderot University, UMR 8257, Paris, France \\ 4 Service de neurologie, HIA Percy, Service de Santé des Armées, Clamart, France \\ 5 Ecole du Val-de-Grâce, Service de Santé des Armées, Paris, France \\ *julien.audiffren@cmla.ens-cachan.fr
} Communicated by Laurent Oudre Demo edited by Julien Audiffren

\begin{abstract}
In this work we discuss the multidimensional scoring approach proposed by Bargiotas and al. [I. Bargiotas, J. Audiffren, N. Vayatis, P-P. Vidal, S. Buffat, A.P. Yelnik and Damien Ricard, On the importance of local dynamics in statokinesigram: a multivariate approach for postural control evaluation in elderly, PloS one, 13 (2018)] which locally characterizes statokinesigrams the trajectory of the center of pressure, which is highly correlated with static balance quality- on small time intervals, or blocks. This approach highlights the local dynamics of the trajectories, and we show that the resulting characterization can be used to provide a global score in order to evaluate the postural control. We evaluate our approach using the statokinesigram of 126 community-dwelling elderly, and show that it provides an efficient tool to discriminate between fallers and non-fallers.
\end{abstract}

\section{Source Code}

The reviewed source code (python3 language) and documentation for this algorithm are available at the web page of this article,${ }^{1}$ together with an online demo. The code is commented in-depth and usage instructions are included in the main.py file of the archive.

Keywords: postural control; center of pressure; statokinesigrams; Gaussian mixture model

\footnotetext{
${ }^{1}$ https://doi.org/10.5201/ipol.2019.251
} 


\section{Introduction}

Postural control is defined as the ability of individuals to maintain a controlled upright position. It is achieved by combination of visual, proprioceptive, and vestibular systems [24]. Impairments and disorders such as myoskeletal disorders, visual, balance or gait impairments throughout age may progressively worsen the individual's postural control, increasing consequently the risk of falling [29]. Falls are considered as one of the major causes of injury in elderly, leading to further restriction in mobility, autonomy problems in daily activities (bathing, cooking, etc.) or even death [20, 30]. Therefore, prevention of falling through prediction and accurate evaluation of risk has become an important issue considering that one third of population $>65$ years-old faces at least one fall per year [30].

A tool of choice for clinical researchers to quantify and evaluate postural control are force platforms. Such platforms record the displacement of the centre of pressure (CoP) which is applied by the whole body in time [27] while the individual stands quietly upon it and follows the clinician's instructions/protocol. This measurement is usually called statokinesigram. Statokinesigrams have been previously used in assessing balance disorder in populations that are either healthy or suffering from balance-related impairments [25, 6]. Many indices derived from the CoP displacement have been showing that $\mathrm{CoP}$ displacement characteristics and dynamic structure (for instance, regularity) can reflect individuals' postural impairment [20, 23, 15, 32, 10, 8]. However, there is no agreement either in healthy or in non-healthy populations $[34,26,14]$ whether these features or transformations alone are able to fully assess the individual's posture control.

In [3], the authors proposed a new approach to exploit the information contained in statokinesigrams, and quantify postural control. The main idea behind this method is twofold. First, the dynamic of the CoP trajectory may change through time; and some part of the statokinesigram of an individual may have "bad postural control profile" (called Unquiet Blocks or UB) while others may have a "good postural control profile" (called Quiet Blocks or QB). Second, the presence, proportion and quality of the $\mathrm{UB} / \mathrm{QB}$ in the statokinesigram are indicative of the quality of postural control. Therefore, identifying QB/UB parts of a signal is key to this method of quantifying static balance.

In order to characterize the local dynamics of statokinesigrams, the authors rely on the following process:

A) the statokinesigram is split into blocks of predefined time-periods (namely 1, 2 or 3 seconds), with fixed overlap (a window starts each 0.5 second);

B) each block is then described with a three dimensional vector, using three well-known and established features from the literature that are known to capture important information about the statokinesigram;

C) each block three dimensional description is then scored, using its probability to belong to the QB and UB clusters;

D) the score of a trajectory is obtained as the average score of all of its blocks.

This approach is tested on a dataset of 126 individuals. Using an standard acquisition protocol inspired by the Romberg test (see e.g. [1]), the authors show that their approach achieves state of the art performance while offering a local, interpretable evaluation of the statokinesigram.

In this paper, we make an in-depth presentation and evaluation of this algorithm, called QUBA (Quiet Unquiet Block decomposition Algorithm) for short, and evaluate it on a new dataset for different tasks. This paper is organized as follows: in Section 2, we describe in detail the scoring algorithm; in Section 3, we detail the learning procedure that was used to obtain the QB/UB clusters, and finally in Section 4 we evaluate the performance of QUBA. 


\section{Description of the Scoring Method}

In this section we describe each step of QUBA in great detail. The algorithm is summarized in Algorithm 1, and each step of QUBA is detailed in the corresponding subsection.

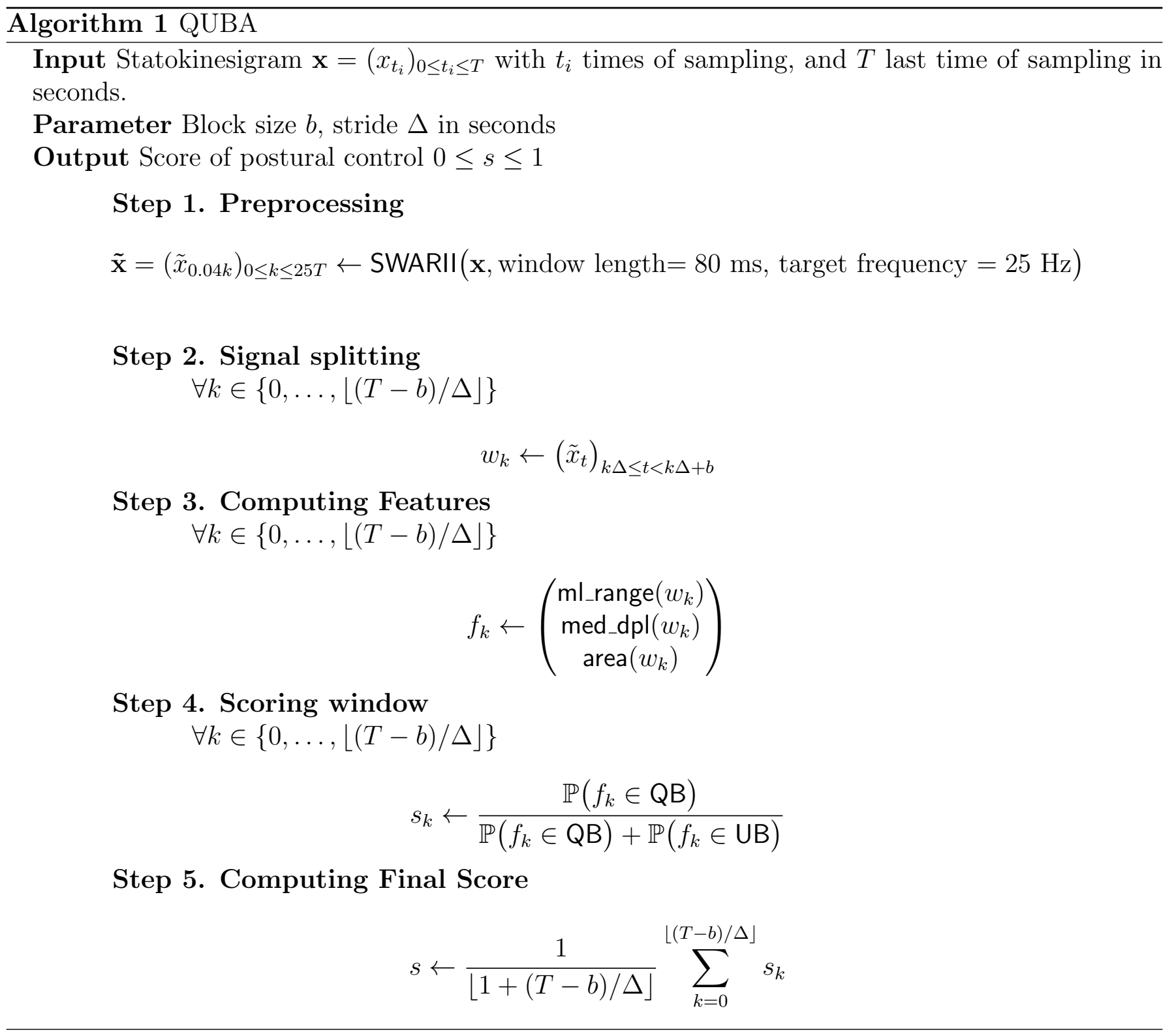

\subsection{Preprocessing of Trajectories}

The first step of the method is the preprocessing of the signals. This part serves the double purpose of A) homogenizing the acquisition frequency and B) denoising the signals. This step is key, as there exist multiple models of force platforms, each with their own characteristics (accuracy, frequency, ... ) [4]. Since the least accurate force platform commonly used in posturography is the Wii Balance Board (WBB) [22], we use its characteristics as the greatest common denominator. As the WBB records the CoP trajectory using a variable time resolution with an average sampling frequency of $60 \mathrm{~Hz}$ [16], we use the the SWARII algorithm [2] for preprocessing, resulting in denoised statokinesigrams uniformly sampled at $25 \mathrm{~Hz} \tilde{\mathbf{x}}$, regardless of the force platform originally used (Step 1). 


\section{$2.2 \quad$ Features}

Signal Splitting. $\tilde{\mathbf{x}}$ is then split in fixed-length time blocks $\left(w_{k}\right)$ of duration $b$, with an step of $\Delta$ between successive blocks (Step 2). The values of $b$ and $\Delta$ that give the best results are $b \in\{1,2,3\}$ and $\Delta=0.5 \mathrm{~s}$.

Feature Computation. A three dimensional representation of each block $w_{k}$, is then computed (Step 3). The three following widely used features [20, 1, 21] are used:

I. ml_range: The range of movement in the Medio-Lateral axis (ML) - the left-right axis. Formally,

$$
\mathrm{ml} \_ \text {range }\left(w_{k}\right)=\max \left\{\tilde{x}_{t}^{\mathrm{ML}} \text { s.t. } \tilde{x}_{t} \in w_{k}\right\}-\min \left\{\tilde{x}_{t}^{\mathrm{ML}} \text { s.t. } \tilde{x}_{t} \in w_{k}\right\}
$$

II. med_spd: The length of the median displacement of the CoP.

$$
\operatorname{med} \_s p d\left(w_{k}\right)=\operatorname{median}\left\{\left\|\tilde{x}_{t+0.04}-\tilde{x}_{t}\right\|_{2} \text {, s.t. } \tilde{x}_{t}, \tilde{x}_{t+0.04} \in w_{k}\right\}
$$

III. area: The surface of the $95 \%$ confidence ellipse $\left(\mathbf{s}_{95}\right)$ of the trajectory of the CoP.

$$
\begin{aligned}
\mu_{k} & \leftarrow \frac{1}{\left|w_{k}\right|} \sum_{\tilde{x}_{t} \in w_{k}} \tilde{x}_{t}, \\
\mathrm{CoV}_{k} & \leftarrow \frac{1}{\left|w_{k}\right|-1} \sum_{\tilde{x}_{t} \in w_{k}}\left(\tilde{x}_{t}-\mu_{k}\right)\left(\tilde{x}_{t}-\mu_{k}\right)^{T}, \\
\operatorname{area}\left(w_{k}\right) & =1.96 \sqrt{\left|\mathrm{CoV}_{k}\right|} .
\end{aligned}
$$

\subsection{Scoring with Gaussian Mixture}

QUBA uses a trained Gaussian Mixture Model (GMM) to predict the probability of the block features $f_{k}$ to belong to the UB (resp. QB) cluster (Step 4). First, each window representation $f_{k}$ is normalized i.e.

$$
\tilde{f}_{k} \leftarrow \frac{f_{k}-\mu}{\sigma},
$$

where $\mu$ and $\sigma$ were learned during the training phase, and the normalization is done element wise. Note that in (1), the normalization is done element-wise, i.e. each feature is normalized separately. This transformation is important as GMM are vulnerable to metric imbalance (see e.g. [33]). Then, for both clusters UB and QB, the probabilities are obtained using the following formula

$$
\mathbb{P}\left(f_{k} \in \mathrm{UB}\right)=\frac{m_{\mathrm{UB}}}{\sqrt{(2 \pi)^{3}\left|\Sigma_{\mathrm{UB}}\right|}} \exp \left(-\frac{1}{2}\left(Z_{j}-\mu_{\mathrm{UB}}\right)^{T} \Sigma_{\mathrm{UB}}^{-1}\left(Z_{j}-\mu_{\mathrm{UB}}\right)\right),
$$

where $m_{\mathrm{UB}}, \mu_{\mathrm{UB}}$ and $\Sigma_{\mathrm{UB}}$ are respectively the mass (relative importance), the mean and the covariance of the UB cluster, and are also learned during the training phase (in our learning process, we constrain the covariance matrix $\Sigma_{\mathrm{UB}}$ to be diagonal). Note that $(2)$ is used to compute $\mathbb{P}\left(f_{k} \in \mathrm{QB}\right)$ by replacing $m_{\mathrm{UB}}, \mu_{\mathrm{UB}}$ and $\Sigma_{\mathrm{UB}}$ by resp. $m_{\mathrm{QB}}, \mu_{\mathrm{QB}}$ and $\Sigma_{\mathrm{QB}}$.

Finally, the score of each $f_{k}$ is obtained as the relative probability of belonging to the QB cluster compared to the UB cluster. The score of the signal is then the average of the score of each window (Step 5). 


\begin{tabular}{l|c|c} 
Variable & Use & Equation \\
\hline$\mu, \sigma$ & Normalization of the feature representation & $(1)$ \\
$m_{\mathrm{UB}}, \mu_{\mathrm{UB}}, \Sigma_{\mathrm{UB}}$ & Probability of belonging to the UB cluster & $(2)$ \\
$m_{\mathrm{QB}}, \mu_{\mathrm{QB}}, \Sigma_{\mathrm{QB}}$ & Probability of belonging to the QB cluster & $(2)$
\end{tabular}

Table 1: Summary of the learned variables used in QUBA. The dependency in $\Delta$ and $b$, and open/closed eyes setting is dropped to keep the notation simple.

\section{Training Process}

Table 1 summarized the variables learned during the training process. Note that these variables interact with the block decomposition of the signal, and therefore depend on the parameters $\Delta$ and b. Additionally, and as discussed in Subsection 3.1, two different models were trained for open eyes and closed eyes statokinesigrams. However, and to ease the reading, these dependencies are kept implicit in the notation.

\subsection{Training Set}

\subsubsection{Demographics}

To train the GMMs, we used the entire dataset considered by [3] in the original paper. Therefore, the training set contained 126 subjects ( $78.5 \pm 7.7$ year-old, 80 females) from the Neurology department of the HIA Percy hospital (Clamart, France) and the consultation office of a practitioner (Paris, France). Inclusion criteria: Participants (1) had age $>65$ years, (2) were addressed in routine consultation in general medicine or neurology, (3) did not suffer from balance related impairment, (4) were able to stand on the platform, (5) gave informed consent. Particularly, only asymptomatic individuals after clinical examination were considered for this study. Individuals which were significantly hypertensive (mean Systolic Blood Pressure (SBP) $\geq 140 \mathrm{mmHg}$ or mean Diastolic Blood Pressure (DBP) $\geq 90$ $\mathrm{mmHg}$ ), hypotensive (SBP $\leq 90 \mathrm{mmHg}$ or $\mathrm{DBP} \leq 60 \mathrm{mmHg}$ ), had particular impairements or used medication which could alter significantly their balance (such as vasoactive, phychotrope drugs) were excluded. Moreover, characteristics such as weight, height and principal syndromes were collected (Table 2). The clinical Research Ethics Committee approved the clinical trial, registered at ANSM (ID RCB 2014-A00222-45).

\begin{tabular}{l|c|c|c} 
& Total Sample & Non-fallers & fallers \\
\hline Demographic & 126 & 108 & 18 \\
\hline Male & 46 & 38 & 8 \\
Female & 80 & 70 & 10 \\
\hline Age (years) & $78.5( \pm 7.7)$ & $77.2( \pm 6.4)$ & $79.2( \pm 7.2)$ \\
Weight (kg) & $69.6( \pm 10.7)$ & $69.4( \pm 10.3)$ & $70.0( \pm 11.4)$ \\
Height $(\mathrm{cm})$ & $167.0( \pm 8.0)$ & $167.0( \pm 8.0)$ & $167.7( \pm 8.3)$ \\
BMI (kg. $\left.m^{-2}\right)$ & $24.96( \pm 2.4)$ & $24.92( \pm 2.3)$ & $25( \pm 2.3)$
\end{tabular}

Table 2: Demographic characteristics of the participants. Fallers are patients who declared at least one fall in the six previous months. No statistically significant difference was found between the two population regarding age, weight, height and body mass index (BMI) $(2$-tailed t-test, $\mathrm{p}$-values $<0.01)$. 


\subsubsection{Balance Measurements}

Balance measurements were acquired using a Wii Balance Board (WBB - Nintendo, Kyoto, Japan). This force platform, while considered a suitable tool for the clinical setting with an acceptable accuracy $[9,16,28]$, is also acknowledged as the least reliable of the force platforms used in research settings [22]. Therefore, QUBA is expected to perform at least as well on data originating from other force platforms (see Section 4). The participants were asked to remove their shoes and step on the platform placing their feet in the most comfortable position without exceeding the shoulder width, and to stand in upright position with open eyes and the arms laying at the side. The trajectory of the CoP positions was recorded for 25 seconds. Subsequently, participants were asked to close their eyes. After a ten-second pause, clinical experts recorded 25 additional seconds with closed eyes. This second acquisition is motivated by findings such as [7], which state that some balance impairments may be more visible in static balance with closed eyes. From each statokinesigram, the first and the last 2.5 seconds were excluded from further analysis (20 seconds per statokinesigram).

\subsubsection{Fall Assessment}

In order to quantify participants balance, a fall questionnaire was filled by each subject keeping information about previous falls during the last six months [17]. Following previous works, participants were classified as fallers if they came to a lower level on the ground unintentionally (at least once in the last six months) [35].

\subsection{Training QUBA}

Parameters. As open eyes and closed eyes trajectories are expected to possess different characteristics, their data were considered separately for training, resulting in two QUBA models. Similarly, and following the results of [3], a different model was trained for each value of $b \in\{1,2,3\}$ seconds. $\Delta$ was set to 0.5 second. In total, six different QUBA models were trained.

Learning normalization variables. First, each signal of the dataset was preprocessed using SWARII (see Subsection 2.1). Then, the resulting resampled statokinesigrams were split in blocks of length $b$, and a three dimensional representation of each time-block was computed (Steps 1-3 of Algorithm 1). After this step, the mean $\mu$ and standard deviation $\sigma$ of each feature over the entire dataset was saved, and used to normalized the training set.

Training the GMM model. We used a two cluster Gaussian Mixture Model (GMM) on the resulting training set. The model was randomly initialized, and trained using the expectation maximization (EM) principle until convergence (i.e. clusters did not change over an iteration). The cluster maximizing the likelihood of the mean of the fallers profiles was labeled as unquiet blocks, and the other as quiet blocks. Examples of the resulting clusters are shown in Figure 1. It is interesting to note that in our experiments, the results were found to be independent of the initialization - the resulting clusters were the same over ten random initializations.

\section{Experimental Evaluation}

Dataset. To evaluate the performance of QUBA, we use the publicly available dataset provided by [11], and we start by briefly describing its content. We would like to emphasize that the version of QUBA used in these experiments was trained on the database of [3] (see Section 3), which is significantly different from this one. The database contains 49 individuals, including 22 elderly 

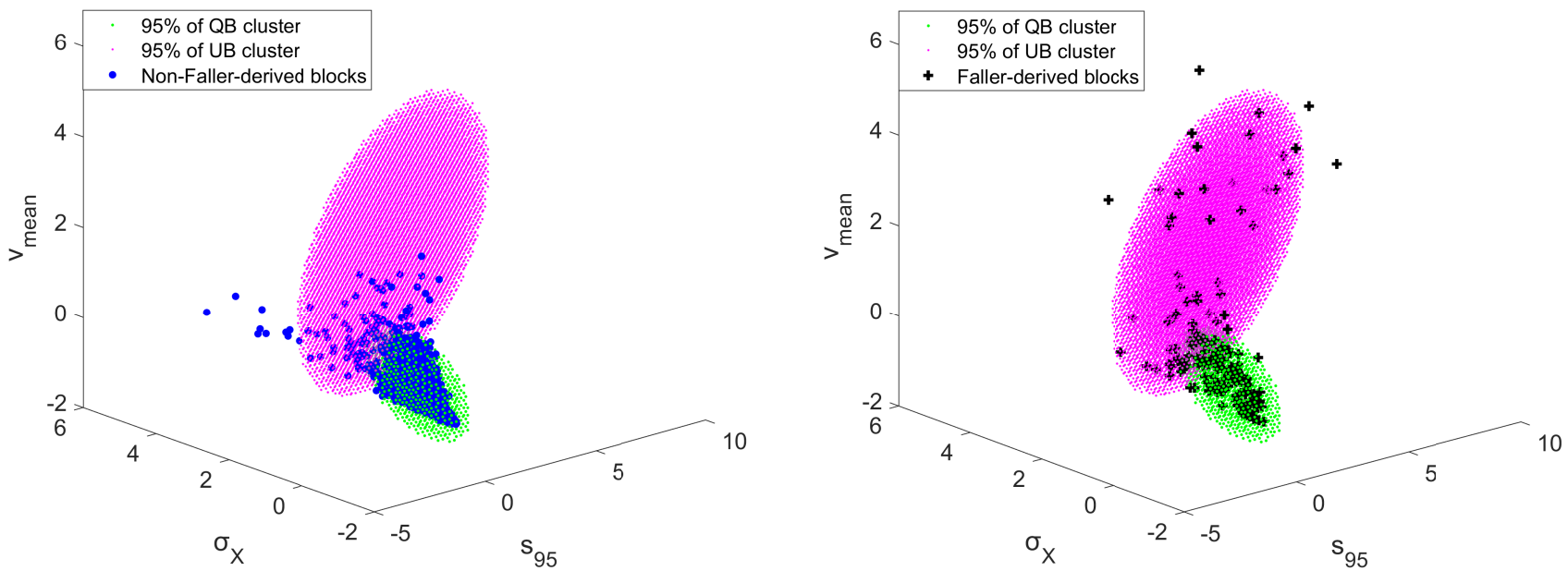

Figure 1: Probability density for the two-component mixture distribution (95\%) and three-second-blocks of A) Non-fallers and $\mathrm{B}$ ) fallers, illustrated in the normalized 3-dimensional space med_spd (v_mean), ml_range $\left(\sigma_{X}\right)$ and area $\left(s_{95}\right)$. The two normal components overlap, but their centers are distinct. Many faller-derived periods are closer to the UB component and conversely, many non-faller derived blocks are closer to the QB centre.

individuals (60 year-old and older) and 27 young individuals (38 year-old and younger), including a large range of postural control profiles (such as disability or disease). Each individual was recorded 12 times, standing still for 60 seconds, under different conditions (open eyes, closed eyes, with or without a foam). Each acquisition was recorded using two OPT400600-1000 AMTI force platforms to track the $\mathrm{CoP}$ position. The dataset also provides the recording of the ground reaction forces and the position of the center of mass, but these data are not used in our analysis. In the following experiments, we use the QUBA algorithm to score and classify different populations under each of the four possible conditions, for each value of the parameter $b \in\{1,2,3\}$

\subsection{Classifying Fallers and Non-Fallers Individuals}

This experiment aims to replicate the result obtained in [3] by classifying Fallers - individuals that have fallen at least once in the past 12 months; total: 132 signals - and Non-Fallers - individuals that have not fallen in the past 12 months; total: 456 signals. The results are presented in Figure 2 and Table 3. The results (AUC) are slightly better than in the original paper in the foam condition, and slightly worse in the rigid condition. Also, the proportion of UB in fallers is greater than in non-fallers, but not significantly so.

\subsection{Classifying Young and Elderly Individuals}

This experiment aims to classify people by age group, as it has been shown that age has a significant impact over postural control (see e.g. [18]). Namely, we aim to separate Elderly individuals - 60 year-old and older; total: 264 signals - and Young individuals - 59 year-old and younger; total: 424 signals. The results are presented in Figure 3 and Table 4 . The results (AUC) are significantly better than the fall related classification in all conditions, and achieves 0.86 AUC in the open eyes foam Surface condition. However, except in this specific set of conditions, the proportion of UB is not statistically different between the two populations. 

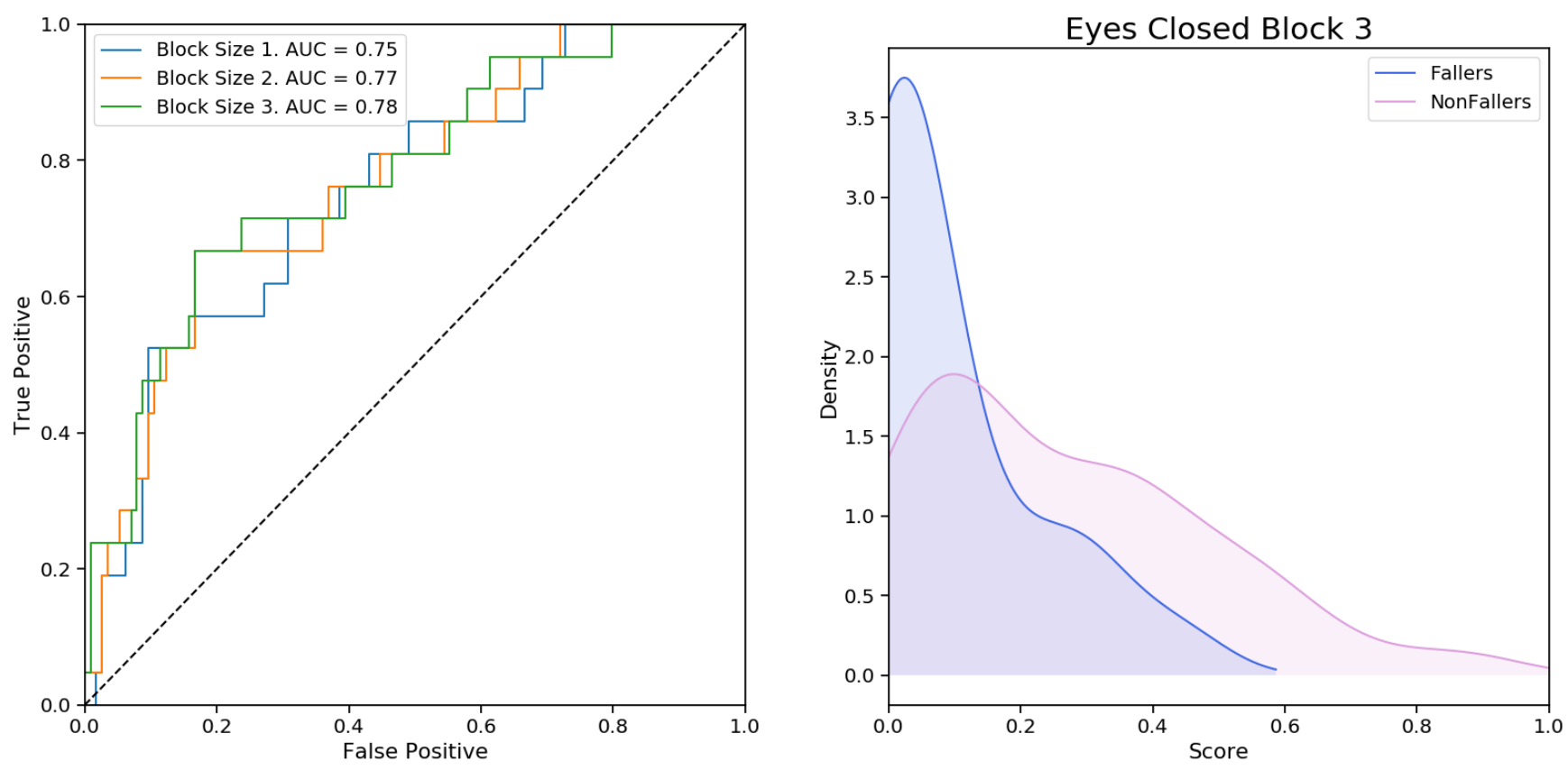

Figure 2: (Left) ROC (Receiving operator Curve) associated with the QUBA algorithm for separating Faller signals and NonFaller signals under the conditions closed eyes and foam. (Right) Estimated distribution (using the kernel approximation method) of the score with $b=3$ among the signals of the Fallers / Non-Fallers under the conditions closed eyes and foam $(A \cup C=0.80)$.

\begin{tabular}{c|c|c|c|c|c} 
Eyes & Surface & BlockSize & AUC & \%UB in Fallers & \%UB in Non-Fallers \\
\hline Open & Rigid & 1 & 0.58 & $0.04( \pm 0.04)$ & $0.03( \pm 0.05)$ \\
Open & Rigid & 2 & 0.60 & $0.05( \pm 0.06)$ & $0.03( \pm 0.06)$ \\
Open & Rigid & 3 & 0.58 & $0.05( \pm 0.07)$ & $0.03( \pm 0.06)$ \\
Open & Foam & 1 & 0.71 & $0.70( \pm 0.17)$ & $0.53( \pm 0.23)$ \\
Open & Foam & 2 & 0.73 & $0.80( \pm 0.15)$ & $0.62( \pm 0.23)$ \\
Open & Foam & 3 & 0.72 & $0.85( \pm 0.14)$ & $0.68( \pm 0.23)$ \\
Closed & Rigid & 1 & 0.56 & $0.03( \pm 0.08)$ & $0.02( \pm 0.04)$ \\
Closed & Rigid & 2 & 0.60 & $0.03( \pm 0.08)$ & $0.02( \pm 0.04)$ \\
Closed & Rigid & 3 & 0.59 & $0.03( \pm 0.08)$ & $0.02( \pm 0.04)$ \\
Closed & Foam & 1 & 0.75 & $0.77( \pm 0.18)$ & $0.57( \pm 0.22)$ \\
Closed & Foam & 2 & 0.77 & $0.86( \pm 0.15)$ & $0.67( \pm 0.22)$ \\
Closed & Foam & 3 & $\mathbf{0 . 7 8}$ & $0.91( \pm 0.13)$ & $0.73( \pm 0.22)$
\end{tabular}

Table 3: AUC values for separating Faller signals and Non-Faller signals, and percentage of UB windows (i.e. windows with score $<0.5)$ among each population for each combination of conditions and value of $b$.

\subsection{Physical Activity}

This experiment aims to classify people by their routine physical activity. Previous works have highlighted the positive impact of physical activity on balance in the elderly (see e.g. [12]). In this experiment, we aim to separate Passive individuals - i.e. they do not practice physical activity regularly: 202 signals - and Active individuals - they practice physical activities at least three times a week, total: 144 signals. The results are presented in Figure 4 and Table 5. The results show no meaningful difference between the two populations, regardless of the conditions. This might be 

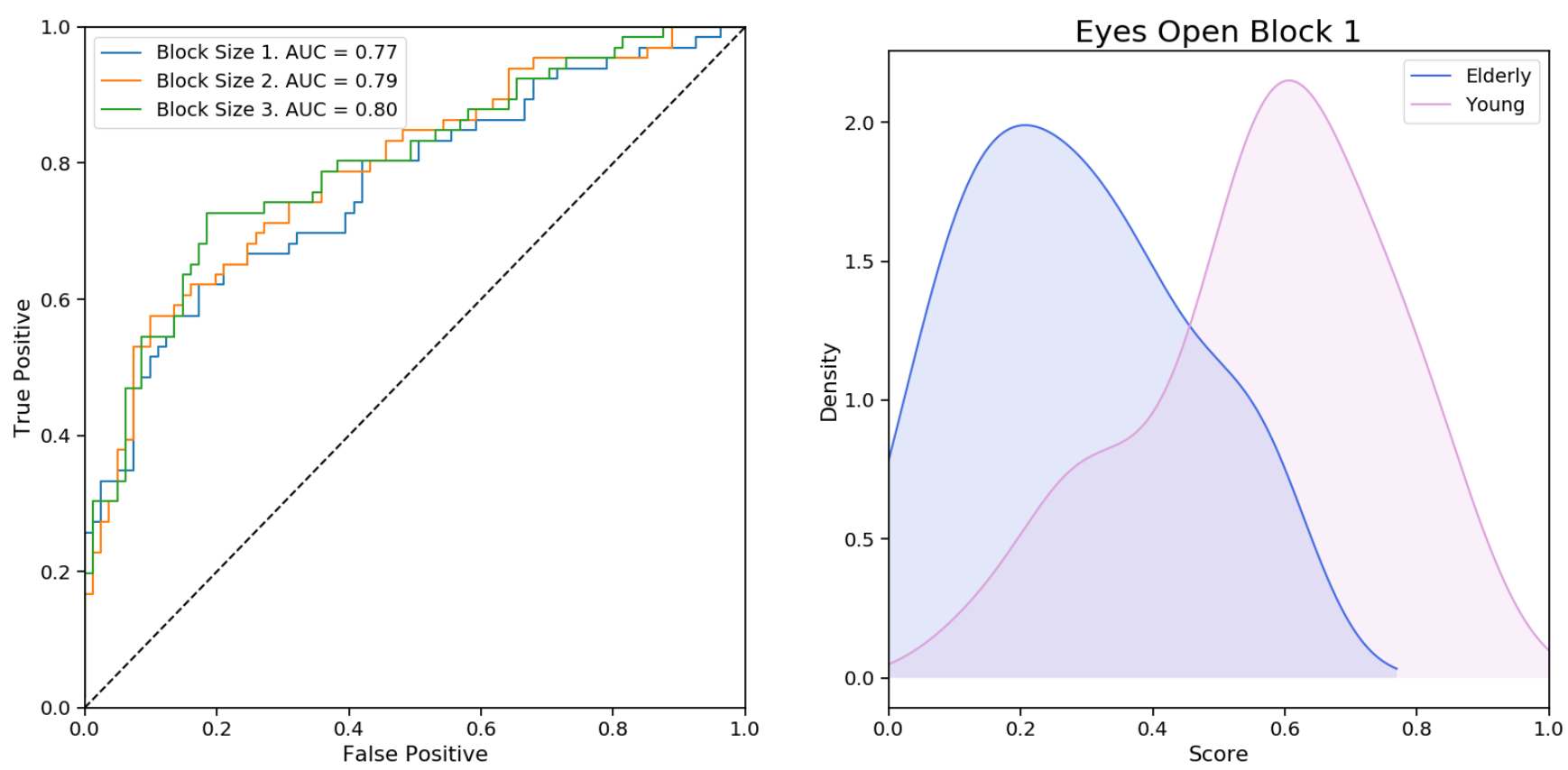

Figure 3: (Left) ROC associated with the QUBA algorithm for separating Elderly and Young signals under the conditions closed eyes and Foam. (Right) Estimated distribution (using the kernel approximation method) of the score with $b=1$ among the signals of the Elderly / Young under the conditions open eyes and Foam $(\mathrm{AUC}=0.86)$.

\begin{tabular}{c|c|c|c|c|c} 
Eyes & Surface & BlockSize & AUC & \%UB in Elderly & \%UB in Young \\
\hline Open & Rigid & 1 & 0.68 & $0.05( \pm 0.07)$ & $0.02( \pm 0.03)$ \\
Open & Rigid & 2 & 0.60 & $0.05( \pm 0.08)$ & $0.02( \pm 0.03)$ \\
Open & Rigid & 3 & 0.56 & $0.05( \pm 0.07)$ & $0.02( \pm 0.03)$ \\
Open & Foam & 1 & $\mathbf{0 . 8 6}$ & $0.71( \pm 0.17)$ & $0.43( \pm 0.19)$ \\
Open & Foam & 2 & $\mathbf{0 . 8 6}$ & $0.81( \pm 0.15)$ & $0.52( \pm 0.20)$ \\
Open & Foam & 3 & $\mathbf{0 . 8 6}$ & $0.86( \pm 0.14)$ & $0.58( \pm 0.21)$ \\
Closed & Rigid & 1 & 0.69 & $0.03( \pm 0.06)$ & $0.01( \pm 0.02)$ \\
Closed & Rigid & 2 & 0.66 & $0.04( \pm 0.07)$ & $0.01( \pm 0.02)$ \\
Closed & Rigid & 3 & 0.64 & $0.03( \pm 0.07)$ & $0.00( \pm 0.02)$ \\
Closed & Foam & 1 & 0.77 & $0.71( \pm 0.21)$ & $0.50( \pm 0.20)$ \\
Closed & Foam & 2 & 0.79 & $0.81( \pm 0.18)$ & $0.60( \pm 0.22)$ \\
Closed & Foam & 3 & 0.80 & $0.87( \pm 0.16)$ & $0.66( \pm 0.23)$
\end{tabular}

Table 4: AUC values for separating Elderly and Young statokinesograms, and percentage of UB windows (i.e. windows with score $<0.5)$ among each population for each combination of conditions and value of $b$

partially explained by the broad definition of physical activity [11], as in this dataset no amount of time or intensity is specified.

\subsection{Surface Type}

Finally, this experiment aims to classify signals depending on their surface condition, Rigid and Foam, as the presence of foam on the force platform significantly alters statokinesigrams. Both populations contained 294 signals, and the results are reported in Figure 5 and Table 6. In this experiment, each condition resulted in an AUC of 1, highlighting the drastic influence of this condition 

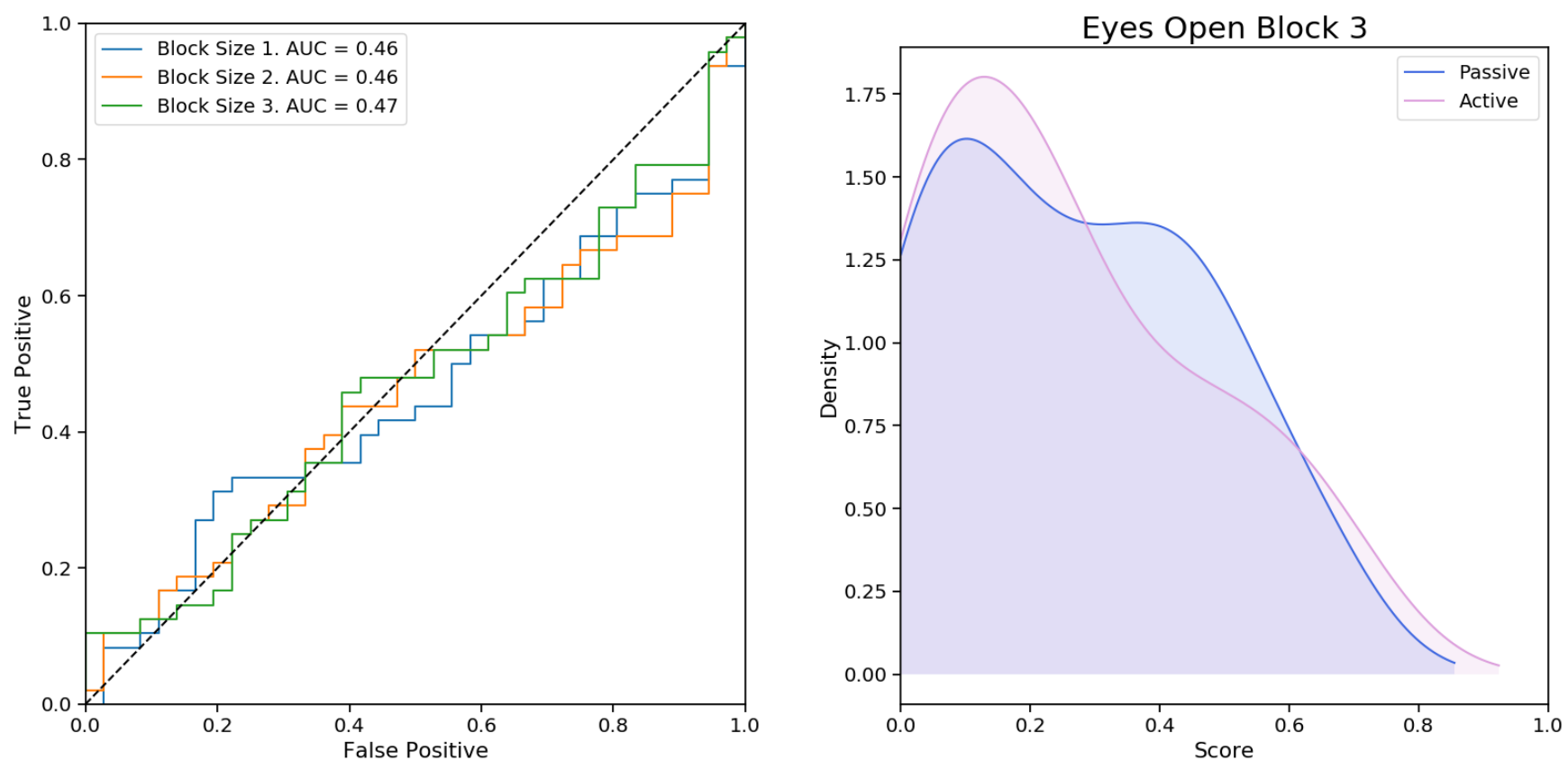

Figure 4: (Left) ROC associated with the QUBA algorithm for separating Passive and Active signals under the conditions closed eyes and Foam. (Right) Estimated distribution (using the kernel approximation method) of the score with $b=3$ among the signals of the Passive / Active under the conditions open eyes and rigid surface (AUC $=0.56)$.

\begin{tabular}{c|c|c|c|c|c} 
Eyes & Surface & BlockSize & AUC & \%UB in Passive & \%UB in Active \\
\hline Open & Rigid & 1 & 0.52 & $0.04( \pm 0.04)$ & $0.05( \pm 0.08)$ \\
Open & Rigid & 2 & 0.54 & $0.04( \pm 0.05)$ & $0.05( \pm 0.09)$ \\
Open & Rigid & 3 & $\mathbf{0 . 5 6}$ & $0.04( \pm 0.06)$ & $0.04( \pm 0.08)$ \\
Open & Foam & 1 & 0.47 & $0.57( \pm 0.21)$ & $0.60( \pm 0.21)$ \\
Open & Foam & 2 & 0.47 & $0.67( \pm 0.21)$ & $0.69( \pm 0.22)$ \\
Open & Foam & 3 & 0.49 & $0.73( \pm 0.20)$ & $0.74( \pm 0.21)$ \\
Closed & Rigid & 1 & 0.50 & $0.03( \pm 0.06)$ & $0.03( \pm 0.05)$ \\
Closed & Rigid & 2 & 0.53 & $0.03( \pm 0.07)$ & $0.03( \pm 0.05)$ \\
Closed & Rigid & 3 & 0.54 & $0.03( \pm 0.07)$ & $0.02( \pm 0.05)$ \\
Closed & Foam & 1 & 0.46 & $0.60( \pm 0.24)$ & $0.63( \pm 0.19)$ \\
Closed & Foam & 2 & 0.46 & $0.70( \pm 0.22)$ & $0.73( \pm 0.18)$ \\
Closed & Foam & 3 & 0.47 & $0.75( \pm 0.21)$ & $0.78( \pm 0.18)$
\end{tabular}

Table 5: AUC values for separating Passive and Active individual signals, and percentage of UB windows (i.e. windows with score $<0.5)$ among each population for each combination of conditions and value of $b$

on postural control.

\section{Discussion}

The objective of this paper was to detail the mechanics of the QUBA algorithm introduced in [3], and to test its behavior on additional datasets. 

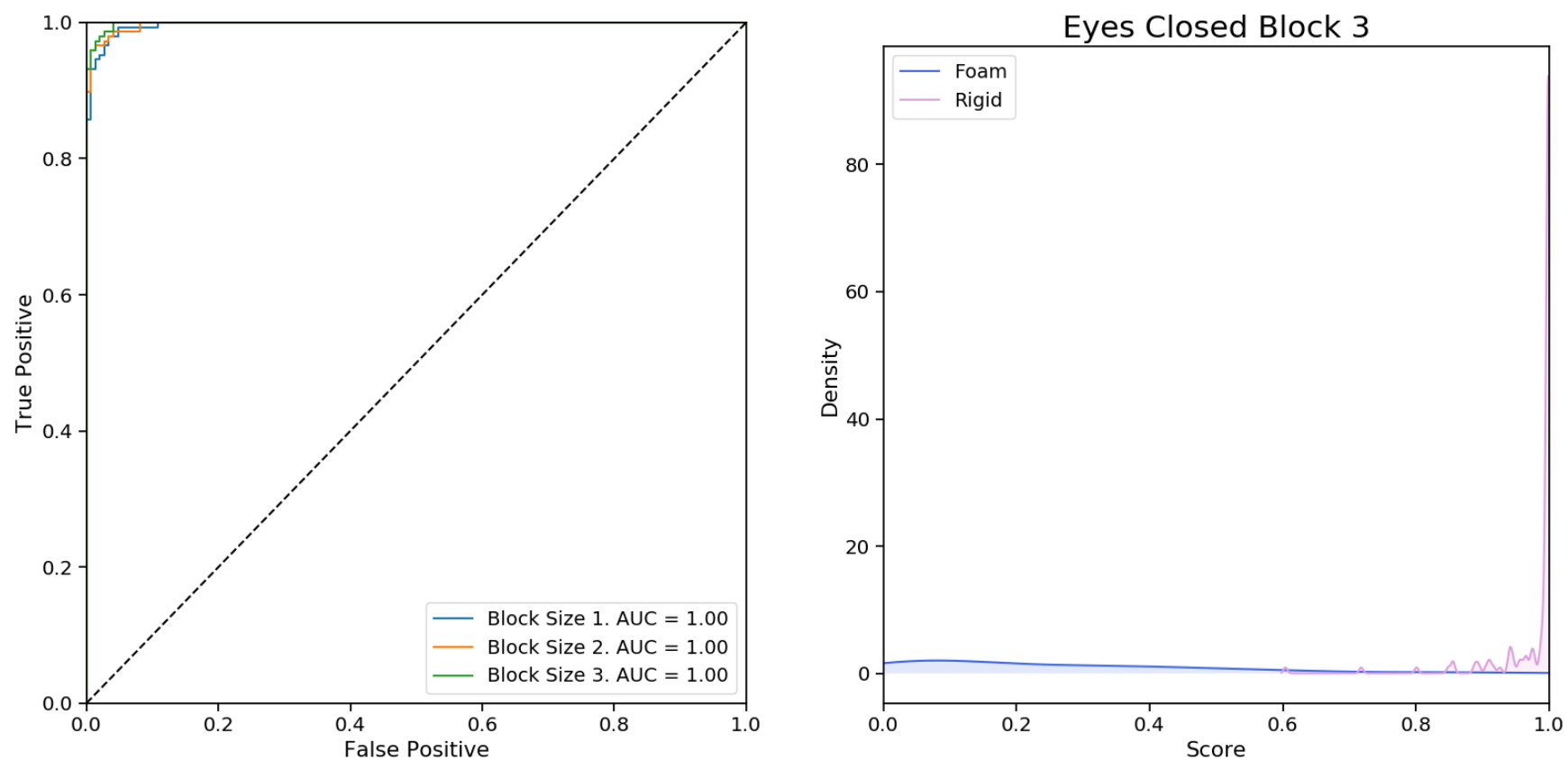

Figure 5: (Left) ROC associated with the QUBA algorithm for separating signals acquired on a Foam/ Rigid surface under the condition closed eyes. (Right) Estimated distribution (using the kernel approximation method) of the score with $b=3$ among the Foam / Rigid signals under the condition closed eyes $(A U C=1)$.

\begin{tabular}{c|c|c|c|c|} 
Eyes & BlockSize & AUC & \%UB in Foam & \%UB in Rigid \\
\hline Open & 1 & 1.00 & $0.56( \pm 0.23)$ & $0.03( \pm 0.05)$ \\
Open & 2 & 1.00 & $0.65( \pm 0.23)$ & $0.03( \pm 0.06)$ \\
Open & 3 & 1.00 & $0.70( \pm 0.23)$ & $0.03( \pm 0.06)$ \\
Closed & 1 & 1.00 & $0.59( \pm 0.23)$ & $0.02( \pm 0.04)$ \\
Closed & 2 & 1.00 & $0.69( \pm 0.23)$ & $0.02( \pm 0.05)$ \\
Closed & 3 & 1.00 & $0.75( \pm 0.23)$ & $0.02( \pm 0.05)$
\end{tabular}

Table 6: AUC values for separating signals acquired on each surface (Rigid and Foam), and percentage of UB windows (i.e. windows with score $<0.5$ ) among each population for each combination of eye condition and value of $b$.

Window representation. QUBA uses a combination of three simple features (Section 2). These features are hardly new, as previous works reported that statokinesigrams medio-lateral and anteroposterior variation, trajectories' velocity, acceleration or CoP's trajectory area [20, 15, 19], might classify CoP trajectories by posture quality. However, and to the best of the authors' knowledge, the local use of these descriptors with QUBA appears to outperform previous attempts at balance quantification by a large margin. This result highlights the benefit of a local analysis of the CoP trajectory, and future works in this direction - such as new custom made descriptors - might significantly improve QUBA.

UB proportion. The wide term "unquiet blocks" might involve periods of transition between strategies, exploration periods, shifting of weight to one foot, head movement or even instability periods that might occur even if subjects are in standing position without disturbance. However, it could be expected that even those periods would be minor in individuals that are able to follow the acquisition protocol and have relatively good postural control. In our experiments, and contrarily to what was observed in [3], in all the rigid surface recording conditions, all individuals appear to have 
little to no UB (Tables 3 and 4). However, the difference between the two populations significantly increased in the foam surface condition. This difference of result might be partially explained by the fact that the threshold of 0.5 for being UB was arbitrary, and might depend on the studied population. It also emphasizes the fact that there is a continuum of type of blocks between UB and $\mathrm{QB}$, and that blocks are rarely completely UB or QB.

Influence of block size. All the tested block lengths $(b=1,2$ or 3 seconds) resulted in near identical performance, with $b=3$ being slightly better than the others. Finding the ideal duration for blocks is difficult, as it is known that age affects drastically (decreases) the sensory response to a small "unstable" moment that may be presented in quiet stand of the elderly. So, a relative slowness in recovery [13] is expected, possibly as "strategy of choice" in order to avoid uncontrolled pass of the stability border [5], and the best value for $b$ may depend on the individual. Therefore, a key direction for improving QUBA would consist in the automatic splitting of the signal into blocks of different length, depending on the CoP dynamics, using for instance rupture detection [31].

QUBA and recording conditions. Tables 3 and 4 show that the best classification results are obtained in the Foam surface condition. Our hypothesis is that the foam placed on the force platform alters postural control, making static balance significantly harder and increasing differences between different qualities of postural control. It also highlights the fact that QUBA score is robust, and can be used in additional settings beyond the one defined in [3].

\section{References}

[1] J. Audiffren, I. Bargiotas, N. Vayatis, P-P. Vidal, And D. Ricard, A non linear scoring approach for evaluating balance: classification of elderly as fallers and non-fallers, PLoS one, 11 (2016), p. e0167456. https://doi.org/10.1371/journal.pone.0167456.

[2] J. Audiffren and E. Contal, Preprocessing the Nintendo Wii board signal to derive more accurate descriptors of statokinesigrams, Sensors, 16 (2016), p. 1208. https://doi.org/10. $3390 / \mathrm{s} 16081208$.

[3] I. Bargiotas, J. Audiffren, N. Vayatis, P-P. Vidal, S. Buffat, A.P. Yelnik, And D. RICARD, On the importance of local dynamics in statokinesigram: A multivariate approach for postural control evaluation in elderly, PloS one, 13 (2018), p. e0192868. https://doi.org/ 10.1371/journal pone.0192868.

[4] H.L. Bartlett, L.H. Ting, and J.T. Bingham, Accuracy of force and center of pressure measures of the Wii balance board, Gait \& Posture, 39 (2014), pp. 224-228. https://doi.org/ $10.1016 / j$.gaitpost.2013.07.010.

[5] J.W. BŁaszczyk and A. Michalski, Ageing and postural stability, Studies in Physical Culture and Tourism, 13 (2006), pp. 11-14.

[6] J.W. BŁaszczyk, R. Orawiec, D. Duda-KŁodowska, and G. Opala, Assessment of postural instability in patients with Parkinson's disease, Experimental Brain Research, 183 (2007), pp. 107-114. https://doi.org/10.1007/s00221-007-1024-y.

[7] M. Bosek, B. Grzegorzewski, A. Kowalczyk, and I. Lubiński, Degradation of postural control system as a consequence of Parkinson's disease and ageing, Neuroscience Letters, 376 (2005), pp. 215-220. https://doi.org/10.1016/j.neulet.2004.11.056. 
[8] J.T. Cavanaugh, K.M. Guskiewicz, and N. Stergiou, A nonlinear dynamic approach for evaluating postural control, Sports Medicine, 35 (2005), pp. 935-950. https://doi.org/10. 2165/00007256-200535110-00002.

[9] R.A. Clark, A.L. Bryant, Y. Pua, P. McCrory, K. Bennell, and M. Hunt, Validity and reliability of the Nintendo Wii balance board for assessment of standing balance, Gait \& Posture, 31 (2010), pp. 307-310. https://doi.org/10.1016/j.gaitpost.2009.11.012.

[10] S.F. Donker, M. Roerdink, A.J. Greven, And P.J. Beek, Regularity of center-ofpressure trajectories depends on the amount of attention invested in postural control, Experimental Brain Research, 181 (2007), pp. 1-11. https://doi.org/10.1007/s00221-007-0905-4.

[11] D.A. Dos Santos, C.A. Fukuchi, R.K. Fukuchi, and M. Duarte, A data set with kinematic and ground reaction forces of human balance, PeerJ, 5 (2017), p. e3626. https: //doi.org/10.7717/peerj.3626.

[12] S. Eyigor, H. Karapolat, B. Durmaz, U. Ibisoglu, and S. Cakir, A randomized controlled trial of Turkish folklore dance on the physical performance, balance, depression and quality of life in older women, Archives of Gerontology and Geriatrics, 48 (2009), pp. 84-88. https://doi.org/10.1016/j.archger.2007.10.008.

[13] F.B. Horak, H.C. Diener, And L.M. Nashner, Influence of central set on human postural responses, Journal of Neurophysiology, 62 (1989), pp. 841-853. https://doi.org/10.1152/jn. 1989.62 .4 .841$.

[14] O. Huxhold, S-C. Li, F. Schmiedek, And U. Lindenberger, Dual-tasking postural control: aging and the effects of cognitive demand in conjunction with focus of attention, Brain Research Bulletin, 69 (2006), pp. 294-305. https://doi.org/10.1016/j.brainresbull.2006. 01.002 .

[15] R.M. Kantner, A.M. Rubin, C.W. Armstrong, and V. Cummings, Force platform balance measures as predictors of indoor and outdoor falls in community-dwelling women aged 63-76 years, American Journal of Otolaryngology, 12 (1991), pp. 196-204. https://doi.org/ 10.1093/gerona/63.2.171.

[16] J.M. Leach, M. Mancini, R.J. Peterka, T.L. Hayes, and F.B. Horak, Validating and calibrating the Nintendo Wii balance board to derive reliable center of pressure measures, Sensors, 14 (2014), pp. 18244-18267. https://doi.org/10.3390/s141018244.

[17] J. Liu, X. Zhang, And T.E. Lockhart, Fall risk assessments based on postural and dynamic stability using inertial measurement unit, Safety and Health at Work, 3 (2012), pp. 192-198. https://doi.org/10.5491/SHAW.2012.3.3.192.

[18] S.R. Lord AND J.A. WARD, Age-associated differences in sensori-motor function and balance in community dwelling women, Age and Ageing, 23 (1994), pp. 452-460. https://doi.org/ 10.1093/ageing/23.6.452.

[19] M. Mancini, A. Salarian, P. Carlson-Kuhta, C. Zampieri, L. King, L. Chiari, AND F.B. HORAK, ISway: a sensitive, valid and reliable measure of postural control, Journal of Neuroengineering and Rehabilitation, 9 (2012), p. 59. https://doi.org/10.1186/ 1743-0003-9-59. 
[20] I. Melzer, N. Benjuya, And J. Kaplanski, Postural stability in the elderly: a comparison between fallers and non-fallers, Age and Ageing, 33 (2004), pp. 602-607. https://doi.org/ 10.1093/ageing/afh218.

[21] J.W. Muir, D.P. Kiel, M. Hannan, J. Magaziner, and C.T. Rubin, Dynamic parameters of balance which correlate to elderly persons with a history of falls, Plos one, 8 (2013), p. e70566. https://doi.org/10.1371/journal.pone.0070566.

[22] G. Pagnacco, E.C.H.W. Oggero, and C.H. Wright, Biomedical instruments versus toys: a preliminary comparison of force platforms and the Nintendo Wii balance board-biomed 2011, Biomedical Sciences Instrumentation, 47 (2010), pp. 12-17.

[23] S. Pajala, P. Era, M. Koskenvuo, J. Kaprio, T. Törmäkangas, and T. Rantanen, Force platform balance measures as predictors of indoor and outdoor falls in community-dwelling women aged 63-76 years, The Journals of Gerontology Series A: Biological Sciences and Medical Sciences, 63 (2008), pp. 171-178. https://doi.org/10.1093/gerona/63.2.171.

[24] R.J. Peterka And M.S. Benolken, Role of somatosensory and vestibular cues in attenuating visually induced human postural sway, Experimental Brain Research, 105 (1995), pp. 101-110. https://doi.org/10.1007/BF00242186.

[25] M. Pirrtola And P. ERA, Force platform measurements as predictors of falls among older people-a review, Gerontology, 52 (2006), pp. 1-16. https://doi.org/10.1159/000089820.

[26] L. Rocchi, L. Chiari, And F.B. Horak, Effects of deep brain stimulation and levodopa on postural sway in Parkinson's disease, Journal of Neurology, Neurosurgery \& Psychiatry, 73 (2002), pp. 267-274. http://dx.doi.org/10.1136/jnnp.73.3.267.

[27] A. Ruhe, R. FeJer, AND B. WALKer, The test-retest reliability of centre of pressure measures in bipedal static task conditions-a systematic review of the literature, Gait \& posture, 32 (2010), pp. 436-445. https://doi.org/10.1016/j.gaitpost.2010.09.012.

[28] P. Scaglioni-Solano and L.F. Aragón-Vargas, Validity and reliability of the Nintendo Wii balance board to assess standing balance and sensory integration in highly functional older adults, International Journal of Rehabilitation Research, 37 (2014), pp. 138-143. https://doi . org/10.1097/MRR. 0000000000000046 .

[29] B. Schoneburg, M. Mancini, F. Horak, and J.G. Nutt, Framework for understanding balance dysfunction in Parkinson's disease, Movement Disorders, 28 (2013), pp. 1474-1482. https://doi.org/10.1002/mds. 25613.

[30] M.E. Tinetti, Preventing falls in elderly persons, New England Journal of Medicine, 348 (2003), pp. 42-49. https://doi.org/10.1056/NEJMcp020719.

[31] C. Truong, L. Oudre, And N. Vayatis, Selective review of offline change point detection methods, arXiv preprint arXiv:1801.00718, (2018). https://arxiv.org/abs/1801.00718.

[32] R.E.A. VAN EMMERIK And E.E.H. VAN WEgEn, On the functional aspects of variability in postural control, Exercise and Sport Sciences Reviews, 30 (2002), pp. 177-183.

[33] D. Ververidis and C. Kotropoulos, Gaussian mixture modeling by exploiting the Mahalanobis distance, IEEE Transactions on Signal Processing, 56 (2008), pp. 2797-2811. https: //doi.org/10.1109/TSP. 2008.917350. 
[34] T. Yamamoto, C.E. Smith, Y. Suzuki, K. Kiyono, T. Tanahashi, S. Sakoda, P. Morasso, AND T. Nomura, Universal and individual characteristics of postural sway during quiet standing in healthy young adults, Physiological Reports, 3 (2015), p. e12329. https://doi.org/10.14814/phy2.12329.

[35] A.A. Zecevic, A.W. Salmoni, M. Speechley, and A.A. Vandervoort, Defining a fall and reasons for falling: comparisons among the views of seniors, health care providers, and the research literature, The Gerontologist, 46 (2006), pp. 367-376. https://doi.org/10.1093/ geront/46.3.367. 